\title{
Existence and Error Estimates for Solutions of a Discrete Analog of Nonlinear Eigenvalue Problems
}

\author{
By R. B. Simpson*
}

\begin{abstract}
Finite-difference methods using the five-point discrete Laplacian and suitable boundary modifications for approximating (1) $-\Delta u=\lambda f(x, u)$ in a plane domain $D, u=0$ on its boundary are considered. It is shown that if (1) has an isolated solution, $u$, then the discrete problem has a solution, $U_{h}$, for which $U_{h}-u=O\left(h^{2}\right)$. If the discrete problem has solutions, $U_{h}$, such that $\left|U_{h}\right| \leqq M$ as $h$ tends to zero, then (1) has a solution, $u$, satisfying $|u| \leqq M$. Let $\lambda^{*}$ be a critical value of $\lambda$ so that (1) has positive solutions for $\lambda \leqq \lambda^{*}$ but not for $\lambda>\lambda^{*}$, then the discrete problem has an analogous critical value $\lambda_{h}^{*}$ and, under suitable conditions, $\lambda_{h}^{*}-\lambda^{*}=O\left(h^{4 / 3-c}\right), \epsilon>0$. Computed results for the case $j(x, u)=e^{u}$ and $D$ the unit square are given.
\end{abstract}

Introduction. For some nonlinear elliptic boundary-value problems, the existence and number of solutions depends strongly on the values of parameters in the problem. In this paper, we present some affirmative answers to the question of whether discrete versions of the problems have the same number of solutions for fixed parameter values; and we establish some asymptotic error estimates for these cases. The problems to be considered are perhaps the simplest ones in which this phenomenon appears nontrivially, the problems of finding solutions, $u(x ; \lambda)$, to the mildly nonlinear problem for a bounded domain $D$ of the plane

$$
\begin{aligned}
-\Delta u(x) & =\lambda f(x, u(x)), & & x \in D, \\
u(x) & =0, & & x \in \partial D .
\end{aligned}
$$

In particular, we are interested in nonlinearities, $f(x, u)$, of the type occurring in certain nonlinear steady-state diffusion problems. For these problems, positive solutions of (0.1), (0.2) are desired and, typically, these exist for certain ranges of $\lambda, 0<$ $\lambda<\lambda^{*}$, where $\lambda^{*}$ and the multiplicity of positive solutions are difficult to predict directly from (0.1), (0.2). A variety of physical problems which can be cast in this form are discussed in "Nonlinear diffusion induced by nonlinear sources" by Joseph and Sparrow [10], along with some analytical techniques for estimating $\lambda^{*}$ and for approximately solving $(0.1),(0.2)$. More extensive bibliographies of literature pertaining to $(0.1),(0.2)$ may be found in [17] and [18].

The dependence of solutions, $u(x ; \lambda)$, of $(0.1),(0.2)$ on $\lambda$ can be summarized by graphs plotting $u(Q ; \lambda)$ versus $\lambda$ for a selected point $Q \in D$; for convenience, in this context, $u(Q ; \lambda)$ will be contracted to $u$. If the geometry of the problem permits, one

Received August 25, 1971.

AMS 1970 subject classifications. Primary 65N10; Secondary 35J60.

Key words and phrases. Finite-difference methods, nonlinear eigenvalue problems, nonlinear elliptic problems, Laplacian, bifurcation, error estimates, Newton method.

* This work was supported by the National Science Foundation under grant NSF GP 16293.

Current address: Department of Applied Analysis and Computer Science, University of Waterloo, Waterloo, Ontario, Canada. 
would naturally choose $Q$ so that $u(Q ; \lambda)=\max _{x}|u(x ; \lambda)|$. For inhomogeneous nonlinearities with $f(x, u)$ convex in $u$, such as $f(x, u)=e^{u}$ or $\left(1+(a u)^{2}\right) u+b$, the $u$ versus $\lambda$ graphs can be expected to resemble the solid line curve in Fig. 1 ([10], [13]) with a maximum at $\left(u^{*}, \lambda^{*}\right)$.

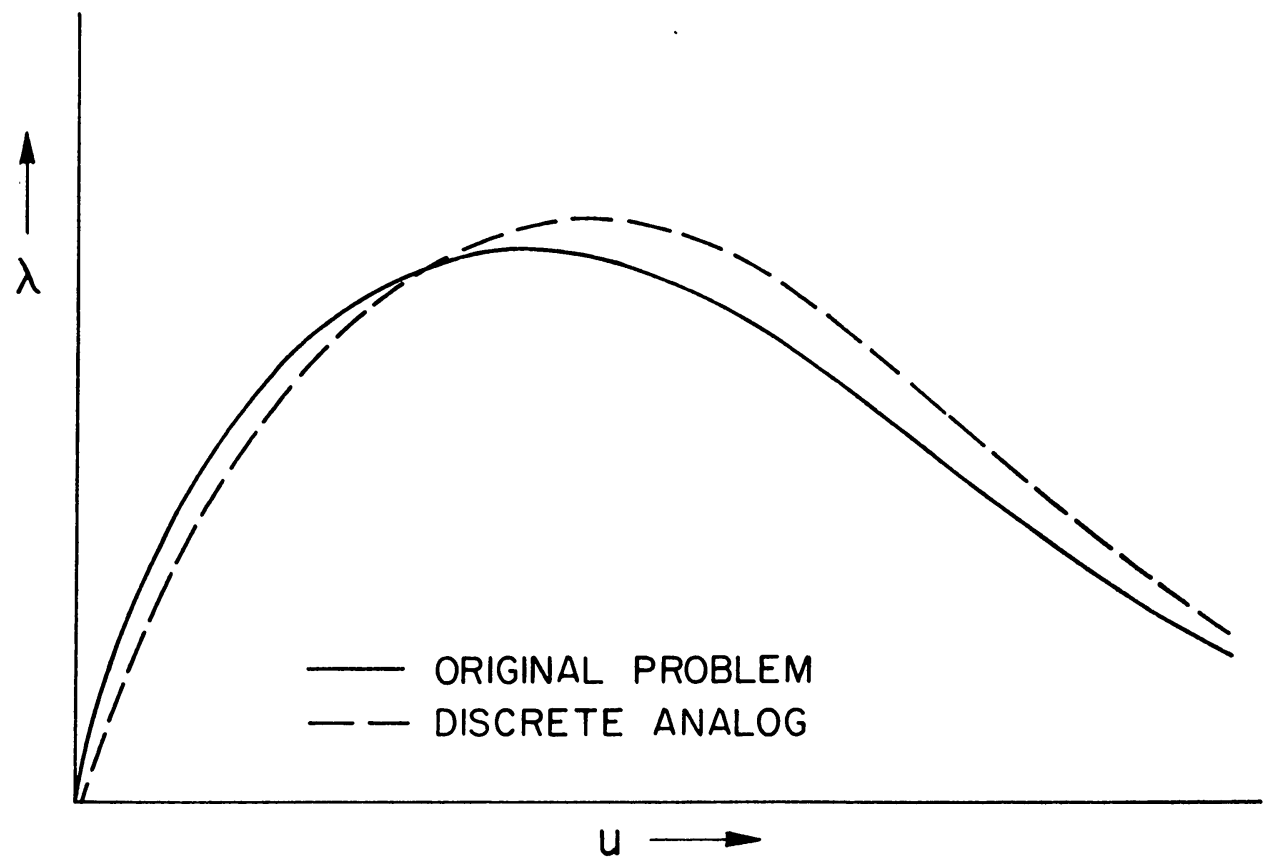

FIGURE 1

The solutions corresponding to the branch of this curve for $u$ between 0 and $u^{*}$ are 'stable' solutions ('stable' in several equivalent senses [6], [13], [18]); however, these stability properties vanish when $u$ reaches and exceeds $u^{*}$.

In this paper, we consider a common finite-difference analog of $(0.1),(0.2)$ and our results prove that the discrete problem has solutions which, when graphed as in Fig. 1 , have basically the same shape as their continuous counterpart, with a maximum of $\left(u_{h}^{*}, \lambda_{h}^{*}\right)$. We show that the graph of discrete solutions converges to that of the continuous problem, with rate $O\left(h^{2}\right)$ away from the maximum (or other extrema) and possibly somewhat slower near the maxima (at least $u_{h}^{*}-u^{*}=O\left(h^{6 / 7}\right)$ and $\lambda_{h}^{*}-$ $\lambda^{*}=O\left(h^{4 / 3-\epsilon}\right)$ for any $\left.\epsilon>0\right)$. In particular, finite-difference methods appear to be a viable approach to calculating $\lambda^{*}$ and $u^{*}$.

A brief outline of the paper is as follows. In Section 1, we give details of our requirements on (0.1) and (0.2); we formulate the discrete problem and note some established properties. In Section 2, we show that if $(0.1),(0.2)$ has a solution (not necessarily stable or positive), then so does its discrete analog and we develop some error estimates for it. We also show that if the discrete problem has solutions with a uniform bound for all $h$ sufficiently small, there must be corresponding solutions of $(0.1)$, (0.2).

In Section 3, we consider the situation graphed in Fig. 1 for positive solutions and prove our assertion about the appearance of the curve of discrete solutions and its 
convergence to that of the continuous case. In Section 4, we review the results of a sample calculation for $f(x, u)=e^{u}$ for the unit square.

1. The Boundary-Value Problem and Discrete Analog. The problem to be considered is posed for a bounded domain, $D$, of the plane, with a piecewise smooth boundary, $\partial D$, and it seeks a function $u(x ; \lambda)$ satisfying (repeated for convenience)

$$
\begin{aligned}
-\Delta u(x ; \lambda) & =\lambda /(x, u(x ; \lambda)), & & x \in D, \\
u(x ; \lambda) & =0, & x & \in \partial D .
\end{aligned}
$$

As basic smoothness requirements on this problem, we assume that $f(x, u)$ is in $C^{2}(\bar{D} \times(-\infty, \infty))$, and that $u(x ; \lambda) \in C^{4}(\bar{D})$. We are primarily interested in nonlinearities in which $f(x, u)$ is monotone increasing in $u$, with $f(x, 0) \geqq 0$; typically, $f(x, u)=e^{u}$ or $u+u^{3}$. We shall require of $f$ that

$$
f_{u}(x, u)>0, \quad(x, u) \in \bar{D} \times(-\infty, \infty) .
$$

Important problems related to $(0.1),(0.2)$ are the linear eigenvalue problems

$$
\begin{aligned}
-\Delta v(x) & =\mu \int_{u}(x, g(x)) v(x), & & x \in D, \\
v(x) & =0, & & x \in \partial D .
\end{aligned}
$$

Let $\mu_{i}(g(x))$ be the eigenvalues of (1.2), (1.3), ordered so that $\mu_{1}(g(x)) \leqq \mu_{i}(g(x))$ for $i>1$.

Definition 1.1. A solution $u(x ; \lambda)$ of $(0.1),(0.2)$, such that $\lambda \neq \mu,(u(x ; \lambda))$ for all $i$, is an isolated solution.

Definition 1.2. A solution $u(x ; \lambda)$ of $(0.1),(0.2)$, such that $\lambda<\mu_{1}(u(x ; \lambda))$, is a stable solution.

In [17], we showed that if $(0.1),(0.2)$ had a positive, stable solution, and a monotone, consistent finite-difference analog was considered, then the discrete problem had a positive solution converging to the positive stable solution. Moreover, if the discretization was strongly stable and was an $O\left(h^{n}\right)$ scheme for linear problems, the convergence to positive stable solutions of this class of nonlinear problems is also $O\left(h^{n}\right)$. In the second section of this paper, we extend these results, for a particular discretization, to hold for any isolated solution of $(0.1),(0.2)$. The particular discretization uses the five-point discrete Laplacian, with the Shortley-Weller modification at mesh points near the boundary. Consider a uniform square mesh of spacing $h$ on the plane and let $D_{h}$ be the set of mesh points lying in $D$. For a mesh point $P$, we shall use $P_{1}, P_{2}$, $P_{3}, P_{4}$ to denote its four nearest neighbors; we define $D_{h}^{*}$ to be the interior mesh points, $C_{h}^{*}$ to be the mesh points near the boundary, and $C_{h}$ to be the intersection of the mesh lines with $\partial D$, i.e.,

$$
D_{h}^{*}=\left\{P \mid P \in D_{h}, P_{i} \in D_{h}, i=1,2,3,4\right\}, \quad C_{h}^{*}=D_{h}-D_{h}^{*} .
$$

If $W(P)$ is a function defined on $D_{h} \cup C_{h}$ and $P \in D_{h}^{*}$, we let

$$
\Delta_{h} W(P)=h^{-2}\left(\sum_{i=1}^{1} W\left(P_{i}\right)-4 W(P)\right) .
$$

If $P \in C_{h}^{*}$, let $P_{1}$ (and $P_{2}$ ), for example, not lie in $D_{h}^{*}$, but let $P_{1}^{*}$ (and $P_{2}^{*}$ ) be the point in $C_{h}$ lying between $P$ and $P_{1}\left(P_{2}\right)$. Let $P_{3}, P$ and $P_{1}^{*}$ lie on a line, with the distance of 
$P_{1}$ to $P$ being $\alpha h(0<\alpha<1)$ (and similarly for $P_{4}, P_{2}$ and $P_{2}^{*}$, the distance $P_{2}^{*}$ to $P$ being $\beta h(0<\beta \leqq 1))$. Then, we define

$$
\begin{aligned}
\Delta_{h} W(P)=2 h^{-2}( & (\alpha+1)^{-1} W\left(P_{3}\right)+(\alpha(\alpha+1))^{-1} W\left(P_{1}^{*}\right) \\
& \left.+(\beta+1)^{-1} W\left(P_{4}\right)+(\beta(\beta+1))^{-1} W\left(P_{2}^{*}\right)-\left(\alpha^{-1}+\beta^{-1}\right) W(P)\right) .
\end{aligned}
$$

For convenience, we assume that if $P \in C_{h}^{*}$, then $P$ has at most two mesh neighbors which are not in $D_{h}$.

The discrete analog then is to solve (1.5), (1.6) for a mesh function $U_{k}(P, \lambda)$ satisfying

$$
\begin{aligned}
-\Delta_{h} U_{h}(P, \lambda) & =\lambda f\left(P, U_{h}(P, \lambda)\right), & & P \in D_{h}, \\
U_{h}(P, \lambda) & =0, & & P \in C_{h} .
\end{aligned}
$$

Discrete analogs of (1.1), (1.2) are the eigenvalue problems

$$
\begin{aligned}
-\Delta_{h} V_{h}(P) & =\mu_{h} f_{u}(P, g(P)) V_{h}(P), & & P \in D_{h}, \\
V_{h}(P) & =0, & & P \in C_{h} .
\end{aligned}
$$

We shall use $\mu_{i, h}(g)$ for the eigenvalues of (1.6), (1.7). When $f_{u}(x, g(x))$ is a smooth positive function, a minor extension of the arguments of [14] show that for each $i$

$$
\left|\mu_{i, h}(g)-\mu_{i}(g)\right|=O\left(h^{2}\right) .
$$

In a paper on error estimates for operators $\Delta_{h}+\lambda I$, Bramble established the a priori inequality (1.10) for the case $f_{u} \equiv 1$ ((5.6) in [4]).

$$
\begin{aligned}
\max _{P \in D_{h} \cup C_{h}}\left|V_{h}(P)\right| \leqq & C\left(\min _{i}\left|\lambda-\mu_{i, h}(u(x ; \lambda))\right|\right)^{-1} \\
& \cdot\left\{\max _{P \in D_{h^{*}}}\left|\left(\Delta_{h}+\lambda f_{u}(P, u(P ; \lambda))\right) V_{h}(P)\right|\right. \\
& \left.+h^{2} \max _{P \in C_{h^{*}}}\left|\left(\Delta_{h}+\lambda f_{u}(P, u)\right) V_{h}(P)\right|+\max _{P \in C_{h}}\left|V_{h}(P)\right|\right\} .
\end{aligned}
$$

The extension of Bramble's result from $f_{u} \equiv 1$ to $f$ satisfying (1.1) is straightforward, using (1.9); so we shall assume both (1.9) and (1.10) in the sequel. Restriction (1.1) on $f(x, u)$ is only used to support our use of (1.9) and (1.10); our results extend to any cases for which these hold.

2. Approximation of Isolated Solutions. In this section, we show that if, for some $\lambda$ value, $(0.1)$ and $(0.2)$ have an isolated solution, then so does its discrete analog for $h$ sufficiently small, and error estimates are given. Moreover, if, for some $\lambda$ value, $(0.1)$ and (0.2) do not have a solution, $u(x, \lambda)$, satisfying $|u(x, \lambda)| \leqq M$, then neither does the discrete problem for $h$ sufficiently small.

THEOREM 2.1. Let $u(x, \lambda)$ be an isolated** solution of $(0.1),(0.2)$ in $C^{4}(\bar{D})$; then, for $h$ sufficiently small, (1.5) and (1.6) have a solution, $U_{h}(P, \lambda)$, for which

$$
\max _{P \in I h}\left|U_{h}(P, \lambda)-u(P ; \lambda)\right|=O\left(h^{2}\right) .
$$

** Definition 1.1. 
Proof. We shall combine the analysis in [12] of the modified Newton (or chord) method for systems of equations with the a priori estimate (1.10) to establish the result. (See also [20], for modified Newton's method.) Let $M F$ denote the vector space of functions defined on $D_{h} \cup C_{h}$ with the norm $\|W(P)\|=\max _{P \in D_{A} \cup C_{A}}|W(P)|$. On this vector space, we define a function, $T$, by prescribing that it map mesh function $W(P)$ into

$$
\begin{aligned}
& T(W)(P)=\Delta_{h} W(P)+\lambda f\left(P, W_{h}(P)\right), \quad P \in D_{h}, \\
& T(W)(P)=W(P), \quad P \in C_{h} .
\end{aligned}
$$

For $T$ so defined, a solution of the discrete problem (1.5), (1.6) is a zero of $T$ and vice versa. Let us denote by $T^{\prime}(W)$, the Fréchet derivative of $T$ formed at $W$ in $M F$. It is straightforward to verify that $T^{\prime}(W)$, operating on $V$ in $M F$, gives

$$
\begin{aligned}
& T^{\prime}(W) V(P)=\Delta_{h} V(P)+\lambda f_{u}(P, W(P)) V(P), \quad P \in D_{h}, \\
& T^{\prime}(W) V(P)=V(P), \quad P \in C_{h} .
\end{aligned}
$$

The modified Newton method for determining zeros of $T$ consists of choosing an initial guess, $U_{h}^{(0)}(P)$, at the solution of (1.5), (1.6) such that

$$
\Gamma=T^{\prime}\left(U_{h}^{(n)}\right)^{-1}
$$

exists and forming the sequence of iterates $U_{h}^{(n)}(P)$ by

$$
U_{h}^{(n)}(P)=U_{h}^{(n-1)}(P)-\Gamma T\left(U_{h}^{(n-1)}(P)\right), \quad n=1,2,3, \cdots .
$$

The success of this method is assured under the following conditions, taken from [12], and in which we use " \|\|$_{M}$ " to denote the induced matrix norm. Let

$$
\begin{array}{r}
\left\|\Gamma\left(T^{\prime}\left(U_{h}^{(1)}\right)-T^{\prime}(W)\right)\right\|_{M} \leqq K\left\|U_{h}^{(0)}-W\right\| \\
\quad \text { for all } W \text { satisfying }\left\|U_{h}^{(0)}-W\right\| \leqq r,
\end{array}
$$

and let $\eta$ be defined by

$$
\eta=\left\|\Gamma\left(T\left(U_{h}^{\prime \prime}\right)\right)\right\|
$$

The conditions are

(i) $\Gamma=T^{\prime}\left(U_{h}^{(0)}\right)^{-1}$ must exist,

(ii) $K r<1 / 4$,

(iii) $\eta<(1-K r) r$,

and when they are met, we can conclude that $T$ has a zero, $U_{h}(P, \lambda)$, satisfying

$$
\left\|U_{h}(P, \lambda)-U_{h}^{(0)}(P, \lambda)\right\| \leqq\left(1-(1-4 K \eta)^{1 / 2}\right) / 2 K .
$$

Let $u(x ; \lambda)$ be an isolated solution of $(0.1),(0.2)$; we shall verify that, for $h$ sufficiently small, we can use $u(x ; \lambda)$ restricted to $D_{h} \cup C_{h}$ as a successful initial guess, $U_{h}^{(0)}(P)$. Since $\mu_{i, h}(u(x ; \lambda))$ converges to $\mu_{i}(u(x ; \lambda)),(1.9)$, as $h$ tends to zero, the assumption that $u(x ; \lambda)$ is isolated implies that, for any $i, \lambda \neq \mu_{i, h}(u(x ; \lambda))$, for $h$ sufficiently small. Consequently, the only solution of

$$
T^{\prime}(u(x ; \lambda)) V(P)=0, \quad P \in D_{h} \cup C_{h},
$$

is $V(P)=0$, and 


$$
\Gamma=T^{\prime}(u(x ; \lambda))^{-1} \text { exists. }
$$

Moreover, there is an $h_{0}$, for which $\min _{i}\left(\left|\lambda-\mu_{i, h}(u(x, \lambda))\right|^{-1}\right)$ is bounded uniformly for $h<h_{0}$, and (1.10) can be interpreted as showing that, for some constant $C$,

$$
\|\Gamma\|_{M} \leqq C \text { for } h<h_{0} .
$$

Let

$$
L(r)=\max \left|f_{u u}(x, \xi(x))\right|,
$$

the maximum taken as $x$ varies over $D$ and $|\xi(x)-u(x ; \lambda)| \leqq r$ for each $x$. From (2.3), it can be seen that for a mesh function $V_{h}(P), T_{h}^{\prime}(u)-T_{h}^{\prime}\left(V_{h}\right)$ is represented by a diagonal matrix

$$
T_{h}^{\prime}(u)-T_{h}^{\prime}\left(V_{h}\right)=\operatorname{diag}\left[f_{u}(P, u(x ; \lambda))-f_{u}\left(P, V_{h}(P)\right)\right]
$$

and so

$$
\left\|\Gamma\left(T_{h}^{\prime}(u)-T_{h}^{\prime}\left(V_{h}\right)\right)\right\|_{M} \leqq\|\Gamma\|_{M} L(r)\left\|u-V_{h}\right\|, \quad \text { if }\left\|u-V_{h}\right\| \leqq r .
$$

Looking at (2.11) and (2.13), we see that here we can set

$$
K=\|\Gamma\|_{M} L(r) \leqq C L(r)
$$

and now turn to determining $\eta$.

In this case. from (2.2),

$$
\begin{aligned}
T_{h}\left(U_{h}^{(n)}\right)(P) & =\Delta_{h} u(P ; \lambda)+f(P, u(P ; \lambda)), & & P \in D_{h}, \\
& =0, & & P \in C_{h}, \\
& =\left(\Delta_{h}-\Delta\right) u(P ; \lambda), & & P \in D_{h},
\end{aligned}
$$

and setting $V_{h}(P)=\Gamma T_{h}\left(U_{h}^{(n)}\right)(P)$ in (1.10), we have, for a constant $\bar{C}$ uniform in $h$,

$$
\begin{aligned}
\eta=\left\|\Gamma T_{h}\left(U_{h}^{(0)}\right)\right\| \leqq \bar{C}\left\{\max _{r^{\circ} \in \nu_{h} h^{*}}\left|\left(\Delta_{h}-\Delta\right) u(P ; \lambda)\right|\right. \\
\left.+h^{2} \max _{P \in C_{h^{*}}}\left|\left(\Delta_{h}-\Delta\right) u(P ; \lambda)\right|\right\} .
\end{aligned}
$$

However, it is well known [3] that if $u(x ; \lambda) \in C^{4}(\bar{D})$, then the first maximum in face brackets in (2.16) is $O\left(h^{2}\right)$ and the second is $O(1)$ for small $h$. Hence, for $\eta$ in (2.7), we have

$$
\eta \leqq M h^{2}
$$

We can now check that conditions (i), (ii), and (iii), following (2.7), are satisfied for $h$ sufficiently small and reach the conclusion stated in the theorem. That (i) is satisfied is stated at (2.10); since $L(r)$ is nondecreasing with $r$, we can choose $r$ small enough to ensure that

$$
K r=C L(r) r<1 / 4
$$

satisfying (ii). With such an $r$, we chose $h_{0}$ small enough that our earlier requirements on this symbol are met and

$$
M h_{0}^{2}<(1-K r) r
$$


Then, evidently (iii) will be satisfied for all $h<h_{0}$, and the existence of a zero $U_{h}(P, \lambda)$ of $T(U)$ is proven. Moreover, from the estimate (2.8) which the modified Newton method affords

$$
\begin{aligned}
\left\|U_{h}(P, \lambda)-u(P, \lambda)\right\| & \leqq\left(1-\left(1-4 K M h^{2}\right)^{1 / 2}\right) / 2 K \\
& =O\left(h^{2}\right) .
\end{aligned}
$$

Hence, the conclusions of the theorem are established.

The use of the analysis of the modified Newton method made in the preceding proof suggests an analogy to the Lax stability convergence equivalence theorem for evolutionary problems. The existence of an isolated solution of the original problem may be compared to the well-posedness requirement of the Lax theorem. Condition (i), following (2.7), and the establishment at (2.11), that \|\|$_{M}$ is bounded uniformly in $h$, is a statement of stability for the (linearized) difference scheme. Looking at (2.7), one can see that a requirement, that $\eta$ tend to zero as $h$ approaches zero, is in effect a consistency requirement on the difference scheme. With these analogies, then, the technique of the proof can be regarded as establishing that for a well-posed problem and a consistent scheme, stability implies convergence (and implies, less trivially here than in the linear case, existence itself).

In the preceding theorem, we considered a fixed $\lambda$; however, in Section 3, we shall allow $\lambda(h)$ to vary with $h$ in such a manner that

$$
\left|\lambda(h)-\mu_{i}(u(x ; \lambda(h)))\right| \geqq O\left(h^{1-\epsilon}\right)
$$

for some fixed $i$ and $-1>\epsilon>0$. If we assume that

$$
\mu_{h, i}(u(x ; \lambda))-\mu_{i}(u(x ; \lambda))=O\left(h^{2}\right)
$$

holds uniformly in $\lambda$, as $\lambda$ varies at least over the range of $\lambda(h)$, then the technique of the preceding proof can be used to establish

COROLlaRY. Let $u(x ; \lambda(h))$ be a family of isolated solutions of $(0.1),(0.2)$ depending on $h$ through $\lambda(h)$, and let (2.21) hold. Then, for $h$ sufficiently small, the discrete problem (1.5) and (1.6) has a family of solutions $U_{h}(P, \lambda(h))$ for which

$$
\max _{P \in D_{h}}\left|U_{h}(P, \lambda(h))-u(P ; \lambda(h))\right|=O\left(h^{1+\epsilon}\right) .
$$

Having shown that there are solutions of the discrete problem converging to each isolated solution of the continuous problem, we now observe that the discrete problem has no additional spurious solutions in the sense of the following theorem.

THEOREM 2.2. Let $h_{i}$ be a sequence of mesh sizes converging to zero. Let $U_{h_{i}}(P, \lambda)$ be a sequence of solutions of (1.5), (1.6) for fixed $\lambda$, with

$$
\max _{P \in D_{n}}\left|U_{h_{i}}(P, \lambda)\right| \leqq M
$$

for some constant $M$. There are extensions $u_{i}(x)$ of $U_{h_{i}}(P)$ to $D$ such that $u_{i}(x)$ is compact in $L_{2}(D)$ and any subsequential limit point, $O(x, \lambda)$, is a solution of $(0.1),(0.2)$ for which $|\bar{O}(x, \lambda)| \leqq M$.

Proof. Let us pick a circle, $K$, with $\bar{D}$ in its interior. The extension, $u_{i}(x)$, of $U_{h_{i}}(P)$ to $K$ is obtained by linearly interpolating $U_{h_{i}}(P)$ for $P \in D_{h}$ and zero at mesh points in $K$, but not in $D_{h}$. Interpolations which accomplish this, for which 


$$
\int_{K}\left(\frac{\partial u_{i}(x)}{\partial x_{1}}\right)^{2}+\left(\frac{\partial u_{i}(x)}{\partial x_{2}}\right)^{2} d x \leqq C h^{2}\left\{\sum_{P \in K}\left(\delta_{1} U_{h_{i}}(P)\right)^{2}+\left(\delta_{2} U_{h_{i}}(P)\right)^{2}\right\},
$$

are described in [2] or [5]. (Here $\delta_{1}, \delta_{2}$ are first forward divided differences in the $x_{1}$ and $x_{2}$ direction.)

In [17, Lemma 3], it is shown that the sum on the right side of (2.24) satisfies

$$
\begin{aligned}
h^{2} \sum\left(\delta_{1} U_{h_{i}}\right)^{2}+\left(\delta_{2} U_{h_{i}}\right)^{2} & \leqq(16 / 15) \sum_{P \in D_{h}} U_{h_{i}}(P)\left(-\Delta_{h} U_{h_{i}}(P)\right) \\
& =(16 / 15) h^{2} \sum_{P \in D_{h}} \lambda U_{h_{i}}(P) f\left(P, U_{h_{i}}(P)\right) .
\end{aligned}
$$

However, since it has been assumed that $\left|U_{h_{i}}(P)\right| \leqq M$, the right side of $(2.25)$ is uniformly bounded in $h_{i}$. Consequently, $u_{i}(x)$ is a bounded sequence in $H_{1}^{0}(D)$ and is compact in $L_{2}(D)$ [1]. Let $u_{i}(x)$ now denote an $L_{2}$ convergent subsequence of the compact sequence; we wish to show that the $L_{2}$-limit, $\bar{u}(x)$, of $u_{i}(x)$ is a solution of $(0.1),(0.2)$. It would seem natural to approach this by first defining a sequence of functions $\gamma_{i}(x)$ by

$$
\begin{aligned}
& -\Delta \gamma_{i}(x)=\lambda f\left(x, u_{i}(x)\right), \quad x \in D, \\
& \gamma_{i}(x)=0, \quad x \in C,
\end{aligned}
$$

and then showing that $\left\{\gamma_{i}(x)\right\}$ and $\left\{u_{i}(x)\right\}$ had the same $L_{2}$-limit by investigating

$$
\Delta_{h} \gamma_{i}(P)-\Delta_{h} u_{i}(P)=\left(\Delta_{h}-\Delta\right) \gamma_{i}(P) .
$$

The author was unable to determine that the $u_{i}(x)$ had enough smoothness (uniformly in $h_{i}$ ) to ensure that (2.27) tends to zero as $h_{i}$ tends to zero. Consequently, a somewhat more involved argument seems necessary. We introduce $W_{i}(P)$ by

$$
\begin{aligned}
\Delta_{h} W_{i}(P) & =\lambda f\left(P, u_{i}(P)\right), & & P \in D_{h}^{*}, * * * \\
W_{i}(P) & =0, & & P \notin D_{h}^{*} .
\end{aligned}
$$

Let $w_{i}(x)$ be the extension of $W_{i}(P)$ to $D$ as piecewise constant over squares of length $h$ centered on the mesh points. Let $G(x ; \xi)$ be the Green's function for the linear version of $(0.1),(0.2)$; we will show that $w_{i}(x)$ converges in $L_{2}$ to a function, $w(x)$, satisfying

$$
w(x)=\lambda \int G(x ; \xi) f(\xi, \bar{u}(\xi)) d \xi .
$$

Then, the proof will be completed by showing that $w_{i}(x)$ and $u_{i}(x)$ have the same $L_{2}$ limit.

Let $\tilde{f}\left(x, u_{i}(x)\right)$ be the piecewise constant extension of $f\left(P, u_{i}(P)\right)$ for $P \in D_{h}^{*}$ and zero for $P \in C_{h}^{*}$ to $D$.

Lemma 2.3. $\tilde{f}\left(x, u_{i}(x)\right)$ converges in $L_{2}(D)$ to $f(x, \bar{u}(x))$.

Proof. This can be established by writing

(2.30) $\tilde{f}\left(x, u_{i}(x)\right)-f(x, \bar{u}(x))=\tilde{f}\left(x, u_{i}(x)\right)-f\left(x, u_{i}(x)\right)+f\left(x, u_{i}(x)\right)-f(x, \bar{u}(x))$

and tackling the terms on the right of (2.30) separately. In the square centered on $P \in D_{h}^{*}$, we have, from the mean value theorem,

\footnotetext{
*** See (1.4) for $D_{h}^{*}$.
} 


$$
\tilde{f}\left(x, u_{i}(x)\right)-f\left(x, u_{i}(x)\right)=\sum_{i=1}^{2}\left\{\frac{\partial f}{\partial x_{i}}(\eta, \theta)+\frac{\partial f}{\partial u}(\eta, \theta) \frac{\partial u_{i}}{\partial x_{i}}(\eta)\right\}\left(x_{i}-P_{i}\right) .
$$

However, since $u_{i}(x)$ is a linear interpolate, either $\partial u_{i} / \partial x_{i}$ vanishes, or it is a first difference of $U_{i}(P)$. Since $|\theta| \leqq M$, one obtains, from integrating over the square and estimating,

$$
\int(\tilde{f}-f)^{2} d x \leqq \kappa\left(\sum_{P \in D} \delta_{1} u_{i}(P)^{2}+\delta_{2} u_{i}(P)^{2}+1\right) h^{4} .
$$

If $P \in C_{h}^{*}$, an additional term of $O(h)$ appears on the right side of $(2.31)$ (i.e., $f\left(P, u_{i}(P)\right)$ $-f(P, 0))$ and from these observations it can be seen that $f\left(x, u_{i}(x)\right)-f\left(x, u_{i}(x)\right)$ converges to zero in $L_{2}(D)$.

From the convergence of $u_{i}(x)$ to $\bar{u}(x)$, the mean value theorem and the uniform boundedness of the $u_{i}(x)$, it follows that $f\left(x, u_{i}(x)\right)$ converges to $f(x, \bar{u}(x))$ in $L_{2}(D)$, completing the proof of the lemma.

Lemma 2.4. $w_{i}(x)$ converges to $w(x)$ defined by $(2.29)$ in $L_{2}(D)$.

Let $G_{h}(P ; Q)$ be the discrete Green's function for the problem (2.28) which defines $W_{i}(P)$ and let $G_{h}(x ; \xi)$ be its piecewise constant extension to $D$. Recalling $w(x)$ from (2.29), we observe that

$$
\begin{aligned}
w_{i}(x)-w(x)= & \int G_{h_{i}}(x, \xi) \tilde{f}\left(\xi, u_{i}(\xi)\right)-G(x, \xi) f(\xi, \bar{u}(\xi)) d \xi \\
= & \int G_{h_{i}}(x, \xi)\left(\tilde{f}\left(\xi, u_{i}(\xi)\right)-f(\xi, \bar{u}(\xi))\right) d \xi \\
& +\int\left(G(x, \xi)-G_{h_{i}}(x, \xi)\right) f(\xi, \bar{u}(\xi)) d \xi .
\end{aligned}
$$

However, from estimates for the discrete Green's function, [2], and Lemma 2.3, we can conclude that the first integral on the right of (2.32) converges to zero in $L_{2}(D)$. Let

$$
y_{i}(x)=\int\left(G(x ; \xi)-G_{h_{i}}(x ; \xi)\right) f(\xi, \bar{u}(\xi)) d \xi
$$

In [2], it is proven that $G_{h_{i}}(x ; \xi)$ converges, as a function of $\xi$, weakly in $L_{p}(D)$ to $G(x ; \xi)$ as $i \rightarrow \infty$. Consequently, $y_{i}(x)$ converges pointwise to zero for $x \in D$. However, $|\bar{u}(\xi)| \leqq M$ ensures that $y_{i}(x)$ is bounded uniformly in $i$ and $x$ and, hence, $y_{i}(x)$ converges to zero in $L_{2}(D)$.

The only task left to us is to show that $u_{i}(x)$ and $w_{i}(x)$ have the same $L_{2}$-limit. From (2.28), we see that

$$
\begin{aligned}
\Delta_{h}\left(W_{i}(P)-U_{i}(P)\right)=0, & P \in D_{h}^{*}, \\
W_{i}(P)-U_{i}(P)=0, & P \in C_{h} .
\end{aligned}
$$

Moreover, since $\Delta_{h} W_{i}(P)$ is bounded for $P \in D_{h}$, uniformly in $h$, standard estimates for $G_{h}(P ; Q)$, [3], show that $W_{i}(P)=O(h)$ if $P$ is a neighbour of a point in $C_{h}^{*}$. Consequently, for the Shortley-Weller operator

$$
\Delta_{h} W_{i}(Q)=O\left(h^{-1}\right) \text { for } Q \in C_{h}^{*} .
$$


Using (2.34) and (2.35) in (1.10) (with $\lambda=0$ ), we see that

$$
\max _{P \in D_{h} \cup r_{h}}\left|W_{i}(P)-U_{i}(P)\right|=O(h) .
$$

However, $\max _{x \in \delta}\left|w_{i}(x)-u_{i}(x)\right|=\max _{P \in D_{A} \cup C_{h}}\left|W_{i}(P)-U_{i}(P)\right|$, since the extensions are piecewise constant and linear. This establishes that $\left\{w_{i}\right\}$ and $\left\{u_{i}\right\}$ have the same $L_{2}$-limit, $\bar{u}(x)$. By (2.29), then

$$
\bar{u}(x)=\lambda \int_{D} G(x, \xi) f(\xi, \bar{u}(\xi)) d \xi
$$

and standard regularity theorems will establish that $\bar{u}(x)$ is a smooth solution.

3. Error Estimates for Discrete Approximations to $u^{*}, \lambda^{*}$. We now specialize our discussion to consider a branch of positive solutions near an extreme point, $\lambda^{*}$, of the range of $\lambda$ for which positive solutions exist. More precisely, we hypothesize that

H1. For a range of $\lambda, \lambda^{*}-a \leqq \lambda<\lambda^{*}$, there are (at least) two positive solutions, $u_{1}(x ; \lambda)$ and $u_{2}(x ; \lambda)$, such that $u_{1}(x ; \lambda)<u_{2}(x ; \lambda), x \in D$.

H2. $u_{1}(x ; \lambda)\left(u_{2}(x ; \lambda)\right)$ is monotone increasing (decreasing) in $\lambda$ for each $x \in D$.

H3. $\lim _{\lambda-\lambda^{*}} u_{i}(x ; \lambda)=u^{*}\left(x, \lambda^{*}\right)$ is a solution of $(0.1),(0.2)$ for $\lambda=\lambda^{*}$.

H4. For a range of $\lambda, \lambda^{*}<\lambda \leqq \lambda^{*}+\delta$, there are no positive solutions of $(0.1)$, (0.2) such that

$$
\max _{x} u(x ; \lambda)<\max _{x} u_{2}\left(x, \lambda^{*}-a\right) \equiv M .
$$

H5. $\mu_{1}\left(u_{2}(x ; \lambda)\right)<\lambda<\lambda^{*}<\mu_{1}\left(u_{1}(x ; \lambda)\right)$ for $\lambda^{*}-a<\lambda<\lambda^{*}$.

These conditions seem to be present in mathematical models of steady-state conditions which are stable for a range of $\lambda$, represented by $u_{1}(x ; \lambda)$, but which become unstable for $\lambda=\lambda^{*}$. While conditions on the problem under which these hypotheses hold in general are not known, a number of problems in which they hold either in part or in full have appeared in the literature. In [13], the minimal positive solution of (0.1), (0.2), with a nonlinearity $f(x, u)$ that is convex in $u$, is shown to satisfy $\mathbf{H} 2$, $\mathrm{H} 3, \mathrm{H} 4, \mathrm{H} 5$. $^{\dagger}$ Much less appears to be known of general behavior of $u_{2}(x ; \lambda)$; however, specific cases are studied in [9], [10], and [11].

Let $\lambda(u)$ and $\lambda_{h}(u)$ denote the functions relating $\lambda$ to $u(Q ; \lambda)$ and $U_{h}(Q ; \lambda)$, respectively, as graphed in Fig. 1 (we assume that $Q$ is a mesh point for every $h$ ). Then, $\lambda^{*}$ is a maximum value for $\lambda(u)$, taken on at $\dot{u}=u^{*}$. It is a straightforward observation on the material in the preceding section that if $\mathrm{H} 1$ through $\mathrm{H} 5$ hold, then, for $h$ sufficiently small, the discrete problem has a critical (maximum) value, $\lambda_{h}^{*}$, which is an analog of $\lambda^{*}$, and, in fact, that

$$
\lim _{h \rightarrow 0} \lambda_{h}^{*}=\lambda^{*} .
$$

Let $\left(u_{h}^{*}, \lambda_{h}^{*}\right)$ be values for which $u_{h}^{*}=U_{h}\left(Q, \lambda_{h}^{*}\right)$; we turn to estimating $\left|\lambda_{h}^{*}-\lambda^{*}\right|$ and $\left|u_{h}^{*}-u^{*}\right|$. We shall assume that the curve for the discrete problem has a unique maximum at $\left(u_{h}^{*}, \lambda_{h}^{*}\right)$ (alternatively, our results could refer to some local maximum $\left(u_{h}^{*}, \lambda_{h}^{*}\right)$ of the discrete curve).

THEOREM 3.1. Suppose that $\lambda(u), \lambda_{h}(u)$ are three times continuously differentiable,

† The claim in [13] that $\mathrm{H} 5$ holds for all minimal positive solutions has been corrected in [15]. 
that $d^{2} \lambda\left(u^{*}\right) / d u^{2}<0$, and that (1.8) holds uniformly for $\lambda^{*}-a<\lambda<\lambda^{*}$ and for both $u_{1}(x ; \lambda)$ and $u_{2}(x ; \lambda)$. Then

$$
\left|u_{h}^{*}-u^{*}\right|=O\left(h^{6 / 7}\right)
$$

and

$$
\left|\lambda_{h}^{*}-\lambda^{*}\right|=O\left(h^{4 / 3-\epsilon}\right)
$$

for any $\epsilon>0$.

Proof. The proof proceeds by replacing the graphs of $\lambda(u)$ and $\lambda_{h}(u)$ by quadratic approximations obtained by interpolation. Estimates for the positions of the actual maxima of these graphs are obtained from the positions of the maxima of the approximating quadratics. For $u$ in the interval $\left[u_{1}\left(Q ; \lambda^{*}-a\right), u_{2}\left(Q ; \lambda^{*}-a\right)\right]$, the graph of $\lambda(u)$ is defined and has the appearance of the solid line in Fig. 2 with a maximum of $\lambda^{*}$ at $u=u^{*}=u^{*}\left(Q ; \lambda^{*}\right)$. Let

$$
\lambda_{i}=\lambda^{*}-i h^{m}, \quad i=1,2,3 \text {, for } 0<m<2 \text { ( } m \text { to be chosen later). }
$$

If we use $u(i)$ to denote $u_{1}\left(Q ; \lambda_{i}\right)$, then since $d^{2} \lambda\left(u^{*}\right) / d u^{2}<0$, we can conclude that

$$
O\left(h^{m / 2}\right)=u(i)-u^{*} \neq o\left(h^{m / 2}\right)
$$

and

$$
O\left(h^{m / 2}\right)=u(i)-u(j) \neq o\left(h^{m / 2}\right) \text { if } i \neq j .
$$

Since $\mu_{1}\left(u_{1}\left(x ; \lambda_{i}\right)\right)>\lambda^{*}(\mathrm{H} 5)$

$$
\mu_{1}\left(u_{1}\left(x ; \lambda_{i}\right)\right)-\lambda_{i} \neq o\left(h^{m}\right)
$$

and so, using the corollary to Theorem 2.1, the discrete problems (1.5) and (1.6), with $\lambda=\lambda_{i}$, will have solutions $U_{h}\left(P ; \lambda_{i}\right)$ for which

$$
U_{h}\left(P ; \lambda_{i}\right)-u_{1}\left(P ; \lambda_{i}\right)=O\left(h^{2-m}\right), \quad P \in D_{h} .
$$

For brevity, we shall write

$$
U_{h}\left(Q ; \lambda_{i}\right) \equiv \bar{u}(i),
$$

so that (3.9), for $P=Q$, could be written

$$
\bar{u}(i)-u(i)=O\left(h^{2-m}\right) .
$$

Let $P(u)$ and $P_{h}(u)$ be quadratics in $u$, interpolating $\left(u(i), \lambda_{i}\right)$ and $\left(\bar{u}(i), \lambda_{i}\right)$, respectively, for $i=1,2,3$. Then, we can write, using divided difference forms,

$$
\begin{aligned}
P(u) & =a(h)(u-u(1))(u-u(2))+b(h)(u-u(1))+u(1), \\
P_{h}(u) & =A(h)(u-\bar{u}(1))(u-\bar{u}(2))+B(h)(u-\bar{u}(1))+\bar{u}(1),
\end{aligned}
$$

where

$$
b(h)=\left(\lambda_{2}-\lambda_{1}\right) /(u(2)-u(1)), \quad B(h)=\left(\lambda_{2}-\lambda_{1}\right) /(\bar{u}(2)-\bar{u}(1)),
$$

and $a(h)$ and $A(h)$ are second divided differences of the interpolation data. Let

$$
v(h)=(u(1)+u(2)) / 2-b(h) /(2 a(h))
$$

be the position of the maximum of $P(u)$ (or minimum if $a(h)>0$ ) and let 
(3.15)

$$
V(h)=(\bar{u}(1)+\bar{u}(2)) / 2-B(h) /(2 A(h))
$$

be the position of the maximum of $P_{h}(u)$. That these actually are maxima will follow from later arguments. Slight modifications of the same arguments produce estimates (3.3) for $u_{h}^{*}-u^{*}$ and (3.4) for $\lambda_{h}^{*}-\lambda^{*}$; the basic arguments depend on the lemmas stated directly below. The arguments giving the estimates follow the statements of the lemmas and the proof of the lemmas follow these. The hypotheses of Theorem 3.1 are assumed for each lemma.

LEMMA 3.2. $v(h)-u^{*}=O\left(h^{3 m / 4}\right)$.

LEMMA 3.3. If $m<4 / 3, V(h)-v(h)=O\left(h^{2-m}\right)$.

LEMMA 3.4. If $m<4 / 3, V(h)-u_{h}^{*}=O\left(h^{3 m / 4}\right)$.

Using these, we can see that

$$
\begin{aligned}
u^{*}-u_{h}^{*} & =u^{*}-v(h)+v(h)-V(h)+V(h)-u_{h}^{*} \\
& =O\left(h^{3 m / 4}\right)+O\left(h^{2-m}\right) .
\end{aligned}
$$

The best result is obtained then when $m=8 / 7$, and the result is (3.3). For (3.4), we see that

$$
\begin{aligned}
\left|\lambda_{h}^{*}-\lambda^{*}\right| & =\left|\lambda_{h}^{*}-\lambda_{1}+\lambda_{1}-\lambda^{*}\right| \\
& \leqq\left|\lambda_{h}\left(u_{h}^{*}\right)-\lambda_{h}(\bar{u}(1))\right|+h^{m} \\
& =O\left(\left|u_{h}^{*}-\bar{u}(1)\right|^{2}\right)+h^{m} .
\end{aligned}
$$

But by (3.16), (3.6) and (3.11)

$$
\begin{aligned}
u_{h}^{*}-\bar{u}(1) & =u_{h}^{*}-u^{*}+u^{*}-u(1)+u(1)-\bar{u}(1) \\
& =O\left(h^{3 m / 4}\right)+O\left(h^{2-m}\right)+O\left(h^{m / 2}\right) .
\end{aligned}
$$

Hence $\left|\lambda_{h}^{*}-\lambda^{*}\right|=O\left(h^{m}\right)+O\left(h^{4-2 m}\right)$.

The best result would be obtained by setting $m=4 / 3$; however, Lemmas 3.3 and 3.4 do not admit this, so (3.4) is obtained by setting $m=4 / 3-\epsilon$ for any $\epsilon>0$.

Proof of Lemma 3.2. The interpolation error at argument $u$ can be expressed as

$$
\lambda(u)-P(u)=\left(\lambda^{(3)}(\xi) / 3 !\right)(u-u(1))(u-u(2))(u-u(3))
$$

for $\xi$ in the interval determined by $u, u(i), i=1,2,3$. Since $u(i)-u^{*}=O\left(h^{m / 2}\right)$, (3.19) shows that the graph of $P(u)$ lies in a strip of vertical height

$$
O\left(\left(\left|u-u^{*}\right|+h^{m / 2}\right)^{3}\right),
$$

centered on the graph of $\lambda(u)$. Hence,

$$
P\left(u^{*}\right) \geqq \lambda^{*}-O\left(h^{3 m / 2}\right) .
$$

Now, suppose $\omega$ varies with $h$ in such a manner that $O\left(h^{m / 2}\right)=\omega-u^{*} \neq O\left(h^{3 m / 4}\right)$, then $\lambda(\omega)<\lambda^{*}-O\left(h^{3 m / 2}\right),{ }^{\dagger \dagger}$ since $d^{2} \lambda\left(u^{*}\right) / d u^{2} \neq 0$, for $h$ sufficiently small. So

$$
P(\omega) \leqq \lambda(\omega)+O\left(\left|\omega-u^{*}\right|+h^{m / 2}\right)^{3}<\lambda^{*}-O\left(h^{3 m / 2}\right) .
$$

Since $P$ has a unique extremum, it must be a maximum and in view of $P\left(v_{h}\right) \geqq P\left(u^{*}\right) \geqq$ $\lambda^{*}-O\left(h^{3 m / 2}\right)$ and (3.21), we can conclude that $v_{h}-u^{*}=O\left(h^{3 m / 4}\right)$. That $\lambda\left(v_{h}\right)-$ $\lambda^{*}=O\left(h^{3 m / 2}\right)$ follows immediately from $d^{2} \lambda\left(u^{*}\right) / d u^{2} \neq 0$.

$$
{ }^{\dagger \dagger}\left(\lambda(\omega)-\lambda^{*}\right) / h^{3 m / 2} \rightarrow-\infty \text { as } h \rightarrow 0 .
$$


Proof of Lemma 3.3. From (3.14) and (3.15),

$$
\begin{aligned}
v(h)- & V(h) \\
& =[(u(1)+u(2))-(\bar{u}(1)+\bar{u}(2))+B(h) / A(h)-b(h) / a(h)] / 2 .
\end{aligned}
$$

Observing (3.11), we conclude that

$$
(u(1)+u(2))-(\bar{u}(1)+\bar{u}(2))=O\left(h^{2-m}\right),
$$

so we consider the remaining difference on the right of (3.22). From (3.7) and (3.11), it can be seen that

$$
O\left(h^{m / 2}\right)=\bar{u}(i)-\bar{u}(j) \neq o\left(h^{m / 2}\right), \quad i \neq j .
$$

So if $m<4 / 3$,

$$
\begin{aligned}
B(h) & =\left(\lambda_{2}-\lambda_{1}\right) /(\bar{u}(2)-\bar{u}(1)) \\
& =b(h)\{(u(2)-u(1)) /(\bar{u}(2)-\bar{u}(1))\} \\
& =b(h)\{1+(u(2)-\bar{u}(2)+\bar{u}(1)-u(1)) /(\bar{u}(2)-\bar{u}(1))\} \\
& =b(h)\left\{1+O\left(h^{2-3 m / 2}\right)\right\} .
\end{aligned}
$$

Turning our attention to the relation between $A(h)$ and $a(h)$, we see that

$$
\begin{aligned}
A(h)= & \left\{\left(\lambda_{3}-\lambda_{2}\right) /(\bar{u}(3)-\bar{u}(2))-\left(\lambda_{2}-\lambda_{1}\right) /(\bar{u}(2)-\bar{u}(1))\right\} /(\bar{u}(3)-\bar{u}(1)) \\
= & \left\{\left(\lambda_{3}-\lambda_{2}\right) /(u(3)-u(2))-\left(\lambda_{2}-\lambda_{1}\right) /(u(2)-u(1))\right\} \\
& \cdot\left(1+O\left(h^{2-3 m / 2}\right)\right) /(u(3)-u(1)) \\
= & a(h)\left(1+O\left(h^{2-3 m / 2}\right)\right) .
\end{aligned}
$$

Using (3.25) and (3.26), we have, since $a(h)$ converges to $d^{2} \lambda\left(u^{*}\right) / d u^{2} \neq 0$,

$$
\begin{aligned}
B(h) / A(h)-b(h) / a(h) & =(b(h) / a(h))\left(1+O\left(h^{2-3 m / 2}\right)\right)-b(h) / a(h) \\
& =(b(h) / a(h)) O\left(h^{2-3 m / 2}\right) .
\end{aligned}
$$

However, by (3.5) and (3.7),

$$
b(h)=\left(\lambda_{2}-\lambda_{1}\right) /(u(2)-u(1))=O\left(h^{m / 2}\right) .
$$

So, from (3.27), we have

$$
B(h) / A(h)-b(h) / a(h)=O\left(h^{2-m}\right)
$$

which, with (3.23), proves the lemma.

Proof of Lemma 3.4. The interpolation error in $P_{h}(u)$ is

$$
\lambda_{h}(u)-P_{h}(u)=\left(d^{3} \lambda_{h}(\xi) / d u^{3}\right)(u-\bar{u}(1))(u-\bar{u}(2))(u-\bar{u}(3)) / 3 ! .
$$

To estimate the size of this error at $u=V_{h}$, we observe from Lemmas 3.2, 3.3 that $v(h)-u^{*}=O\left(h^{3 m / 4}\right), V_{h}-v(h)=O\left(h^{2-m}\right)$ and from (3.6) and (3.11) that $u(i)-$ $u^{*}=O\left(h^{m / 2}\right)$ and $u(i)-\bar{u}(i)=O\left(h^{2-m}\right)$. Consequently,

$$
\vec{u}(i)-V(h)=O\left(h^{m / 2}\right), \quad i=1,2,3,
$$


and, from (3.30), the interpolation error at $u=V(h)$ is $O\left(h^{3 m / 2}\right)$. Now, if $V_{h}^{+}=$ $V_{h}+h^{m / 2}$ and $V_{h}^{-}=V_{h}-h^{m / 2}, O\left(h^{m}\right)=P\left(V_{h}^{ \pm}\right)-P\left(V_{h}\right) \neq o\left(h^{m}\right)$, since $A(h) \neq 0$ for $h$ sufficiently small. However, since the interpolation error at $u=V_{h}^{ \pm}$is $O\left(h^{3 m / 2}\right)$, we will have

$$
\lambda\left(V_{h}^{ \pm}\right)<\lambda\left(V_{h}\right)
$$

for $h$ sufficiently small. Hence, $u_{h}^{*}$ must lie between $V_{h}^{-}$and $V_{h}^{+}$and $V_{h}-u_{h}^{*}=O\left(h^{m / 2}\right)$. (Here, we use the assumption that $\lambda_{h}(u)$ has one maximum at $u_{h}^{*}$; otherwise, as mentioned above, the result will be valid for some local maximum, call it $u_{h}^{*}$, of $\lambda_{h}(u)$.)

Now, if $u$ varies with $h$ so that $O\left(h^{m / 2}\right)=u-V_{h} \neq O\left(h^{3 m / 4}\right)$, then $P\left(V_{h}\right)-$ $P(u)>O\left(h^{3 m / 2}\right)$. But

$$
P\left(V_{h}\right)-\lambda_{h}\left(V_{h}\right)=O\left(h^{3 m / 2}\right)
$$

and

$$
P(u)-\lambda_{h}(u)=O\left(h^{3 m / 2}\right),
$$

so $\lambda_{h}(u)<\lambda_{h}\left(V_{h}\right)$ for $h$ sufficiently small. Consequently, $u \neq u_{h}^{*}$ and $V_{h}-u_{h}^{*}=$ $O\left(h^{3 m / 4}\right)$ as stated. It would appear that improved estimates could be obtained by this method by interpolating to higher degrees and assuming additional smoothness of the $\lambda(u), \lambda_{h}(u)$ curves; however, the analysis becomes overly clumsy and the author believes an alternative mode of analysis should be sought to obtain such results.

4. An Example. The preceding theoretical results were tested on the problem

$$
\begin{aligned}
-\Delta u(x) & =\lambda e^{u(x)}, & & x \in D, \\
u(x) & =0, & & x \in \partial D,
\end{aligned}
$$

for $D$ taken to be the unit square. It has been conjectured that, for this problem, there is a $\lambda(u)$ curve with a single maximum. To the author's knowledge, however, there are no existence proofs for any solutions of this problem with $\lambda>0$, except when $D$ is a circle, in consequence of the very strong convex nonlinearity. (See [6] and [7] for case of $D$ a circle.) If, however, solutions exist, the arguments of Keller and Cohen, [13], show that the minimal positive solution is stable, and that other solutions are not stable. Recently, Joseph and Sparrow, [10], have given a simple analytic technique for estimating the $\lambda(u)$ curve, at least up to its first maximum. Discrete approximations to solutions of this problem (and other mildly nonlinear problems) have been computed by Rosen, [19], with error bounds, by nonlinear programming techniques. It appears that the bounding technique presented in this paper is restricted to stable solutions of (0.1), (0.2), as defined in Definition 1.2.

In the discrete equations, for (4.1), (4.2), the distinction between stable and unstable solutions of (4.1), (4.2) is reflected in the fact that the Jacobian matrix of the equations will be symmetric but not positive definite at discrete solutions corresponding to unstable continuous solutions. For such solutions, regular gradient-type iterative processes, such as nonlinear versions of SOR, cannot be expected to converge. On the other hand, Newton's method, implemented by solving directly a linear system at each step, is known to converge if the above-mentioned Jacobian matrix is simply nonsingular, [12]. This latter property holds, for $h$ sufficiently small, at least, for dis- 
crete solutions which correspond to isolated solutions of (4.1), (4.2). For this reason, the discrete equations were solved using Newton's method, solving at each step a linear system directly by a block tridiagonal scheme [8]. ${ }^{\dagger \dagger}$ The discrete problem was posed (using symmetry assumptions) for a quarter of the square, and mesh sizes $h=.1$ and $h=.05$. The results were consistent to two or three decimals and are presented in Fig. 2 and Table 1, along with the bound from [10] for comparison.

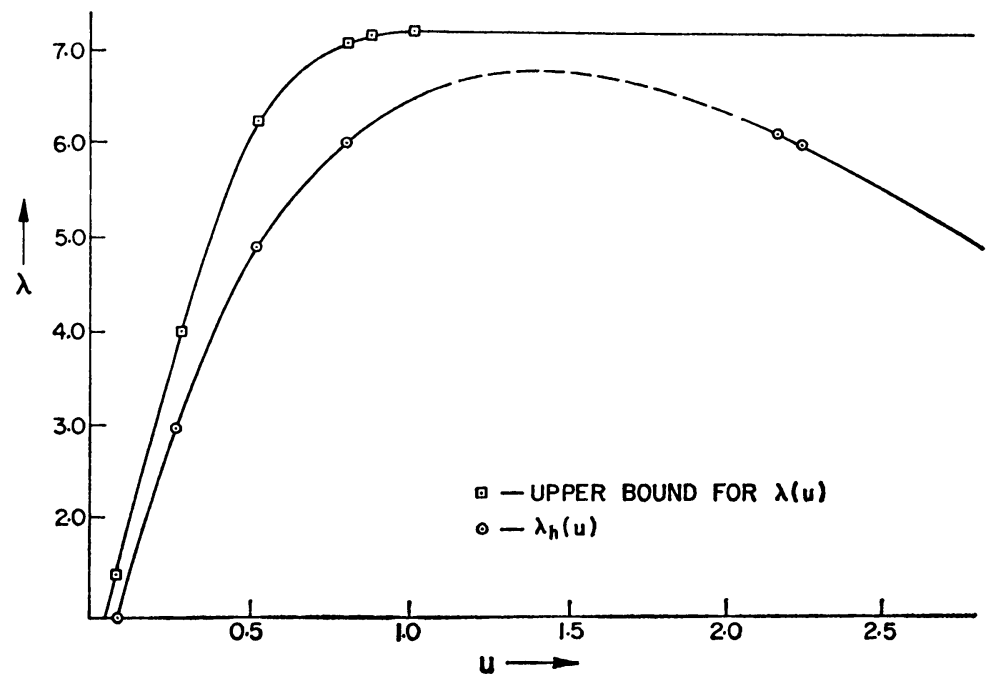

FIGURE 2

TABLE 1

\begin{tabular}{cccc}
\hline$u$ & $\lambda_{h}(u)$ & bound for $\lambda(u)$ \\
\hline .078 & 1.0 & 1.43 \\
.269 & 3.0 & 4.06 \\
.555 & 5.0 & 6.29 \\
.796 & 6.0 & 7.10 \\
.867 & 6.2 & 7.20 \\
1.006 & 6.5 & 7.26 \\
1.072 & 6.6 & 7.26 \\
1.163 & 6.7 & 7.26 \\
2.161 & 6.1 & \\
2.228 & 6.0 & \\
2.846 & 5.0 & \\
4.231 & 3.0 & \\
6.982 & 1.0 & \\
\hline
\end{tabular}

tIt The calculation was implemented by Mrs. M. Brown during the progress of her thesis on calculation of saddle points and the author gratefully acknowledges her work. 
The Jacobian matrix of the discrete system is the matrix for the discrete operator $-\Delta_{h}+\lambda f_{u}(P, W(P))$ of (1.7), (1.8), which is singular for $\lambda$ replaced by $\mu_{i, h}(W(P))$. Since $\lambda-\mu_{1, h}\left(u_{h}(x ; \lambda)\right)$ tends to zero as $\lambda$ approaches $\lambda_{h}^{*}$, it would be anticipated that the discrete problem would be increasingly difficult to solve as $\lambda$ nears $\lambda_{h}^{*}$, and this was indeed the case. Moreover, if we let $\bar{U}_{h}(P ; \lambda)$ and $\underline{U}_{h}(P ; \lambda)$ denote the upper and lower solutions, respectively, the arguments of [13] indicate that $\mu_{1, h}\left(D_{h}(P ; \lambda)\right)<$ $\lambda<\lambda_{h}^{*}<\mu_{1, h}\left(\underline{U}_{h}(P ; \lambda)\right)$ so that, for a given $\lambda$ value near $\lambda_{h}^{*}$, the Jacobian matrix at the upper solution might be expected to be more singular than the Jacobian matrix at the lower solution. This also was encountered in the sense that, for $\lambda$ near $\lambda_{h}^{*}$, there are a number of $\lambda$ values for which initial guesses for Newton's method producing convergence to the lower solution were obtained but we were unable to produce convergence to the upper solution.

The bound from [10] for this case is

$$
\begin{aligned}
\lambda(u) & \leqq 2 \pi^{2} u e^{-u}, & & u \leqq 1, \\
& \leqq 2 \pi^{2} e^{-1}, & & u \geqq 1,
\end{aligned}
$$

and the results are consistent with Rosen's estimate of $\gamma^{*}=6.81$ [19].

Department of Mathematics

Rensselaer Polytechnic Institute

Troy, New York 12181

1. S. Agmon, Lectures on Elliptic Boundary Value Problems, Van Nostrand Math. Studies, no. 2, Van Nostrand, Princeton, N. J., 1965. MR 31 \#2504.

2. J. H. BRAMBLE, "On the convergence of difference approximations to weak solutions of Dirichlet's problem," Numer. Math., v. 13, 1969, pp. 101-111. MR 40 \#3729.

3. J. H. Bramble, \& B. E. HubBaRD, "On the formulation of finite difference analogues of the Dirichlet problem for Poisson's equation," Numer. Math., v. 4, 1962, pp. 313-327. MR 26 \#7157.

4. J. H. BRamble, "Error estimates for difference methods in forced vibration problems," SIAM J. Numer. Anal., v. 3, 1966, pp. 1-12. MR 34 \#969.

5. K. O. Friedrichs \& H. B. Keller, A Finite Difference Scheme for Generalized Neumann Problems, Numerical Solution of Partial Differential Equations (Proc. Sympos. Univ. Maryland, 1965), Academic Press, New York, 1966, pp. 1-19. MR 34 \# 3803.

6. H. FuJita, "On the nonlinear equations $\Delta u+e^{u}=0$ and $\partial v / \partial t=\Delta v+e^{v}, " B u l l$. Amer. Math. Soc., v. 75, 1969, pp. 132-135. MR 39 \#615.

7. I. M. GEL'FAND, "Some problems in the theory of quasi-linear equations," Uspehi Mat. Nauk, v. 14, 1959, no. 2 (86), pp. 87-158; English transl., Amer. Math. Soc. Transl., (2), v. 29, 1963, pp. 295-381. MR 22 \#1736; MR 27 \% 3921 .

8. E. IsaACSON \& H. B. Keller, Analysis of Numerical Methods, Wiley, New York, 1966. MR 34 \#924.

9. D. D. JOSEPH, "Nonlinear heat generation and stability of the temperature distribution in conduction solids," Int. J. Heat Mass Transfer, v. 8, 1965, pp. 281-288.

10. D. D. JOSEPH \& E. M. SPARROw, "Nonlinear diffusion induced by nonlinear sources," Quart. Appl. Math., v. 28, 1970, pp. 327-342.

11. H. B. KELLER, "Elliptic boundary value problems suggested by nonlinear diffusion processes," Arch. Rational Mech. Anal., v. 35, 1969, pp. 363-381. MR 41 \#639.

12. H. B. KelleR, "Newton's method under mild differentiability conditions," J. Comput. System Sci., v. 4, 1970, pp. 15-28. MR 40 \#3710.

13. H. B. KelleR \& D. S. COHEN, "Some positone problems suggested by nonlinear heat generation," J. Math. Mech., v. 16, 1967, pp. 1361-1376. MR 35 \#4552.

14. J. R. KUTTLER, "Finite difference approximations of eigenvalues of uniformly elliptic operators," SIAM J. Numer. Anal., v. 7, 1970, pp. 206-232. MR 42 \#8717.

15. T. LAETSCH, "A note on a paper of Keller and Cohen," J. Math. Mech., v. 18, 1968/ 69, pp. 1095-1100. MR 40 \#1716. 
16. JA. G. Panovko \& I. I. Gubanov, Stability and Oscillations of Elastic Systems. Paradoxes, Fallacies, and Concepts, "Nauka", Moscow, 1964; English transl., Consultants Bureau, New York, 1965. MR 32 \#4914; MR 33 \#2025.

17. R. B. Simpson, "Finite difference methods for mildly nonlinear eigenvalue problems," SIAM J. Numer. Anal., v. 8, 1971, pp. 190-211.

18. R. B. SIMPSON \& D. S. CoHEN, "Positive solutions of nonlinear elliptic eigenvalue problems," J. Math. Mech., v. 19, 1970, pp. 895-910.

19. J. B. Rosen, "Approximate solution and error bounds for quasi-linear elliptic boundary value problems," SIAM J. Numer. Anal., v. 7, 1970, pp. 80-103. MR 41 \#9452.

20. L. V. Kantorovič \& G. AkIlov, Functional Analysis in Normed Spaces, Fizmatgiz, Moscow, 1959; English transl., Internat. Series of Monographs in Pure and Appl. Math., vol. 46, Macmillan, New York, 1964. MR 22 \#9837; MR 35 \#4699. 